\title{
Tiempo, tarea y satisfacción. De la sindéresis a la medición de resultados
}

\author{
Time, Task, and Satisfaction. From \\ Synderesis to Results Measurement
}

\author{
JUAN ANDRÉS MERCADO \\ Pontificia Università della Santa Croce (Roma). \\ mercado@pusc.it \\ ORCID: 0000-0001-7016-9045
}

Resumen: Polo explica la sindéresis como un principio proactivo. Para ser constructiva, la efusividad humana requiere disciplina individual y estructuras organizativas. Una relación armónica entre la inteligencia y las emociones constituye una plataforma para la esperanza y la gratificación. Las emociones indican que las acciones y las producciones positivas producen satisfacción y perfeccionan a los trabajadores. Los directivos deben promover actividades que lleven a cumplir los objetivos. Fijar objetivos estructura el tiempo a través de instrumentos muy variados, para que los trabajadores puedan dar más y seguir dando, es decir, sin desgastarse innecesariamente. La metodología OKR concreta algunas nociones antropológicas fundamentales de Polo, a través del establecimiento de objetivos y estrategias para medir los resultados de modo que los trabajadores crezcan en autonomía e iniciativa. La retroalimentación que se da con este sistema de trabajo promueve la confianza y la asimilación de las gratificaciones por los resultados alcanzados.

Palabras clave: Leonardo Polo, Gestión Del Tiempo, Crecimiento Humano, Sindéresis

\section{Abstract:}

Polo explains synderesis as a proactive principle. Human effusiveness needs personal discipline and formal organisation to be constructive. A harmonic relationship between intelligence and emotions constitutes a platform for hope and gratification. Feelings indicate that positive activities and good productions bring satisfaction and perfect the working person. Managers must exercise and promote activities to realise the company's goals. Goal-setting structures human time with a wide variety of means so that people can give more and keep on giving, i.e. without wearing themselves out unnecessarily. The OKR methodology concretises some of Polo's fundamental anthropological notions. Objective setting and instruments to measure results support workers' autonomy and initiative. The feedback given with this work system promotes trust and the internalisation of the gratifications for the results achieved.

Key words: Leonardo Polo, Time Management, Human Flourishing, Synderesis

* Agradezco a la Redacción de Empresa y Humanismo y a los evaluadores las sugerencias que he procurado incorporar en esta nueva versión del texto. 


\section{INTRODUCCIÓN}

Leonardo Polo explicó en distintas ocasiones temas de gran calado de su propuesta antropológica para públicos no especializados en filosofía y en cursos de iniciación filosófica para estudiantes universitarios. Este estudio aprovecha abundantes pasajes de los escritos producidos a partir de esas lecciones, para asociarlos a algunas ideas de la psicología contemporánea y de la literatura sobre el management.

El hilo conductor es que la capacidad humana de dar, es decir, la efusividad de la persona humana, que se manifiesta ya en la sindéresis, necesita disciplina por parte del individuo y estructura por parte de las organizaciones. La disciplina, que está ligada a la necesidad de organizarse según la inteligencia, se ve también como una plataforma de esperanza y gratificación. Las pasiones o emociones indican que lo que se hace bien produce satisfacciones y perfecciona a los participantes del trabajo compartido. Estas dos relaciones, es decir, la que media entre la capacidad de donar y la sindéresis, y la que conecta disciplina y gratificación, se ilustrarán a lo largo de estas páginas.

Los responsables en las organizaciones deben ejercer y promover las actividades en vistas a la realización de los objetivos de la empresa. Eso supone estructurar el tiempo con distintos medios, para que las personas puedan dar más y seguir dando, es decir, sin desgastarse innecesariamente.

La claridad de la comunicación al establecer tareas y objetivos, la medición de los resultados y la retroalimentación son fundamentales en las organizaciones, y es mejor compartirlos que guardarlos celosamente. La dirección y el liderazgo se basan en un sistema de crecimiento de los participantes, no en la personalidad de un líder.

En la última parte del texto se presenta el sistema de trabajo basado en los objetivos y los resultados clave (OKR) como una vía de estructuración del tiempo humano en las organizaciones. La metodología OKR es compatible con la propuesta de Polo y, como es obvio, decididamente más concreta e incisiva en los detalles.

\section{LA SINDÉRESIS}

La interpretación que hace Polo de la sindéresis tiene un marcado carácter exhortativo. Al clásico primer principio del actuar, que frecuentemente se resume como "hacer el bien y evitar el mal", el filósofo español da una colora- 
ción positiva, que podríamos llamar proactiva: haz el bien, actúa ${ }^{1}$. La persona se puede cansar, pero sabe que su obligación es seguir, que debe seguir haciendo el bien:

"actúa todo lo que puedas y mejora tu actuación. El mal, ya se sabe, está prohibido. Evitar el mal es un no, pero la negación no es lo primero en la moral. El conocimiento moral de principios impulsa, ratifica que el hombre debe tener iniciativa. No es un deber añadido, sino la expansión de la libertad"2.

Esta exhortación a perseguir el bien, a efectuarlo, se extiende a todo el arco de la vida. Se trata de elegir continuamente hacer el bien, sin retraerse. El perno de la actividad es la actitud de la persona "ante la larga tarea que es vivir, ante el proyecto humano que es desarrollar su existir incrementando lo real”. La persona se sabe llamada a aportar, a no quedarse corta ${ }^{3}$.

Por eso, Polo entiende este resorte fundamental de la actividad como la relación que se descubre entre la propia situación y una realidad que exige iniciativa. Más que de una norma, se trata de:

"la conexión de cualquier norma conmigo, pues la norma moral no es una instancia obligatoria que se yerga ante mí solitaria como un reclamado cumplimiento forzado"

Esta especie de insatisfacción constructiva está hondamente enraizada en la antropología trascendental poliana, que desarrolla ampliamente las cuestiones ligadas a la capacidad de dar del ser humano. Es también un elemento característico de la antropología cristiana ${ }^{5}$. Ese no querer detenerse es el primer principio de la esperanza, y depende de lo que Polo llama la libre destinación o destinarse libre. La esperanza como manifestación de la donación es modesta, pues:

"no reclama la autoría del otorgamiento ni exige su reconocimiento: renuncia a que sobre ella recaiga la atención ajena, precisamente porque no renuncia a dar, y porque la insatisfacción equivale a no cansarse de dar"6.

\footnotetext{
Cfr. Polo, L. (2018), pp. 279-280.

2 Polo, L. (2018), p. 280. Véase también Polo, L. (2016b), p. 312, donde se glosa el célebre "si dices basta pereciste", de San Agustín.

3 Cfr. Polo, L. (2018), p. 280.

4 Polo, L. (2018), p. 280.

5 Cfr. Benedicto XVI (2005).

6 Polo, L. (2019), p. 234.
} 


\section{LA ÉTICA COMO ORGANIZACIÓN DE LA EFUSIVIDAD}

Por su índole social, la persona humana se desarrolla en comunicación con los demás, y su crecimiento se da siempre en compañía. La configuración de la vida humana en las organizaciones modernas adquiere dimensiones particulares en las cuales es preciso armonizar la producción de objetos, la adaptación de sistemas, la generación de soluciones formativas, con la exigencia de ayudar a crecer a todos los participantes. Esto supone una sofisticada armonización de las tendencias y las facultades cognoscitivas.

Es evidente que la sensibilidad se queda en lo inmediato y por eso carece de la perspectiva del futuro. Por eso el orden de las elecciones y de las actividades no puede depender de ella. Es decir, no se le puede asignar un papel estructurador, que depende de un nivel más elevado de conocimiento, capaz de ordenar?. Esa estructuración es éticamente relevante porque "la ética desde el punto de vista temporal es la organización de la biografía humana", para la cual no basta la satisfacción de solicitaciones inmediatas, como las del conocimiento sensible.

A la inteligencia corresponde sopesar las elecciones en vista de fines a mediano y largo plazo. Por otro lado, como ninguno de los fines alcanzados o alcanzables es definitivo, la persona debe ser capaz de gestionar esa tensión hacia los bienes de manera coherente para seguir creciendo?

La visión de la inteligencia permite además la coordinación con los demás, y reglamentarla, es decir, la división del trabajo, que es necesaria y fundamental para las empresas. Es vital articular tanto la diversidad de cualidades personales -aptitudes de cada uno para distintas actividades-, como la de las facultades -todos tenemos distintas capacidades a desarrollar: inteligencia, voluntad, imaginación, habilidad técnica y manual- ${ }^{10}$.

El conocimiento intelectual le permite a la persona substraerse al flujo temporal, para poder jerarquizar los bienes y los objetivos. Se trata, como indica Polo, de una biperteleologización, que se corresponde con las modalidades

7 Cfr. Polo, L. (2019), p. 386. Este pasaje se inserta en el detallado tratamiento del mando, hasta la p. 395.

8 Polo, L. (2019), p. 271.

9 Cfr. Polo, L. (2019), p. 271. Sobre la necesidad de las virtudes para esa estructuración, cfr. las pp. 244-245.

10 Cfr. Polo, L. (2019), pp. 366-367. Son las primeras páginas de "Hacia un mundo más humano". Véase también Baumeister, R. F. (2005), pp. 33-34, 386. Sobre la importancia de la división del trabajo, cfr. Baumeister, R. F., y Bushman, B. J. (2017), pp. 47, 493. 
de la libertad, o sea, con un dominio de sí que se manifiesta en la libertad de elección (libertad de) y la libertad para desarrollarse (libertad para $)^{11}$.

Polo señala que para hacer fructificar esta capacidad se requieren hábitos firmes, como la fortaleza, para pechar con las exigencias de los planes de largo plazo, lo cual tiene que ver con lo que él llama el primer teorema de los agentes del cambio:

"una institución funciona bien si tiene objetivos a largo plazo, persiguiendo los cuales, la institución crece y es el agente de un cambio, porque también es capaz de transformar medios en fines"12.

Más adelante explicaré algunas conexiones entre la percepción sensible (las emociones positivas) y el mantenimiento de la tensión en los proyectos. Ahora me interesa señalar una coincidencia importante entre el tratamiento del filósofo español sobre la voluntad, con los estudios de Roy Baumeister.

\section{LAS CAPACIDADES HUMANAS EN CONJUNTO}

Es evidente que, en la base del orden entre las potencias, que permite el crecimiento, está la fuerza de voluntad o autocontrol. Esta capacidad de contenerse permite elegir, moderar y dirigir las acciones ${ }^{13}$.

Antes de mencionar algunas coincidencias temáticas importantes del trabajo de psicólogos sociales como Baumeister (1953) y Tomasello (1950), me parece importante subrayar un aspecto de su aproximación a la consideración de las características de los seres humanos, que los hacen capaces de comportarse de manera peculiar. Ambos autores sostienen una versión de la evolución de los primates hasta el ser humano en la que es evidente la continuidad biológica. Sin embargo, les resulta claro que hay una serie de factores elementales que, en un momento dado, hace que uno de los homínidos se distancie netamente de los demás. Es como si el surgimiento de una capacidad se diera simultáneamente con el desarrollo de otras, cuya conjunción le permite em-

11 Cfr. Polo, L. (2019), pp. 371; 378-379.

12 Polo, L. (2019), p. 378.

13 "Una acción que surge del conocimiento, y un conocimiento que suspende la acción, van más allá de lo puramente animal, porque en el animal el conocimiento está incrustado en la dinámica natural [...] el conocimiento humano no es una fase de la conducta sino una suspensión de ella" (Polo, L. y Llano, C. (2019), pp. 322-323). Cfr. Baumeister, R. F. (2005), pp. 36-37. Cfr. Mercado, J. A. (2021), pp. 130139: ahí se presentan algunos pasajes fundamentales de Platón y Aristóteles sobre el "descubrimiento" de la capacidad de elección y su papel en la formación del carácter. 
pezar a construir su historia, sin depender como antes de los factores naturales.

Baumeister cita la formulación del ratchet effect ("efecto de retén" o "de escalada" o "de trinquete") de Michael Tomasello"14. La idea proviene de los sistemas de bloqueo de los engranajes, y el término es usual en economía. En biología se usa para ilustrar cambios en los que, una vez alcanzada una cierta condición, se va hacia adelante sin retroceder; es una capacidad restrictiva de los procesos humanos en los cuales, una vez acaecido un evento, no se da involución, sino un progreso en la complejidad de las actividades.

Siguiendo las conclusiones de Tomasello y su equipo, Baumeister explica los dos elementos que se necesitan para esa progresión abierta solamente hacia adelante: la primera es la innovación: alguien aporta una solución mejor a un problema compartido ${ }^{15}$. La otra es la conservación: la solución se transmite a otros y se recuerda, de modo que todos pueden seguir utilizándola incluso tras la desaparición del inventor. Tomasello afirma que los simios no humanos demuestran una notable habilidad para encontrar soluciones novedosas y creativas a los problemas. Lo que no consiguen es la conservación y transmisión de ese saber. A pesar de que el innovador recuerde la solución durante un tiempo, y un par de sus compañeros la copien y la usen durante un tiempo, finalmente se olvida, y la siguiente generación vuelve al punto de partida ${ }^{16}$.

Se requeriría espacio abundante para conectar esta visión de la cultura como ventaja competitiva de los seres humanos, con la propuesta antropológica de Polo. Entre las nociones del filósofo que cabe conectar con estas tesis está la descripción del capital como técnica. Me limito por ahora a subrayar la idea del capital como descubrimiento de una técnica o saber hacer con la cual se usan mejor los instrumentos o se crean otros nuevos ${ }^{17}$. Es una transformación integradora de los bienes de producción y "un medio capaz de una continuación inventiva especialmente intensa" que tiende a la expansión ${ }^{18}$.

14 Cfr. Tomasello, M. (2019), pp. 4, 19 y c. 5, y Baumeister, R. F. (2005), p. 31. La aplicación de esta noción por parte de Baumeister está muy ligada a la fuerza de voluntad entendida como autocontrol. Durante años, Baumeister colaboró con Walter Mischel en la explicación de las conexiones de esta cualidad humana con las otras notas distintivas que lo hacen capaz de crear su propio mundo, es decir, la cultura.

15 Cfr. Baumeister, R. F. (2005), pp. 31-36.

16 Cfr. Tomasello, M. (2019), pp. 3-9; 134-135.

17 Cfr. Polo, L. (2019), p. 271, "El capital como técnica".

18 Polo, L. (2019), p. 136, "El capital y el tiempo. El crecimiento". 
A lo largo de estas páginas subrayaré otras coincidencias significativas. Ahora me interesa destacar un aspecto metodológico.

La psicología exige una comprensión de conjunto, una captación de correlaciones que va más allá del análisis ${ }^{19}$, para entender los organismos. Este tipo de conocimiento crece entendiendo la unidad en la complejidad ${ }^{20}$, es decir, la coherencia de los sistemas ${ }^{21}$. Según Polo, la inducción en el pensamiento aristotélico apunta en esta dirección, no solamente al establecimiento de universalizaciones de tipo estadístico ${ }^{22}$.

Esa comprensión es correlativa con la apertura y la indeterminación, es decir, con la inventiva ${ }^{23}$. Tal indeterminación se conjuga con la apertura de la inteligencia y la multiplicidad de necesidades. La apertura permite al ser humano no solamente generar sistemas ordenando cosas -como en el uso de instrumentos- sino también organizarse con otros "porque nunca hace aisladamente: hace con alguna cosa y para otra cosa; es decir que al hacer con las cosas las vincula; por eso, el hombre añade al mundo su propia organización”"24.

\section{ALEGRÍA Y ESPERANZA}

La esperanza de conseguir algo es un elemento fundamental del motor que mantiene vivo el dinamismo de cualquier programa o plan de acción.

Aristóteles describía la esperanza entre otras pasiones de los seres racionales. Este sentimiento se desarrolla a partir de la orientación que se da a las

19 Baumeister destaca frecuentemente las correlaciones entre las características anatómicas y las capacidades de movimiento; entre la potencia de la memoria humana y la comprensión y elaboración de significados, etc.

20 Cfr. Polo, L. (2016b), pp. 162-163.

21 Cfr. Polo, L. (2016b), pp. 29 y ss., 164. Una comparación entre el método analítico y el sistémico se encuentra en esa misma obra: "El método adecuado para conocer la esencia del hombre", pp. 159168; Polo, L. (2018), pp. 43-57, "Las limitaciones del método analítico”; Polo, L., (2016a), c. 2; Polo, L. y Llano, C. (2019), c. 1. Polo reflexionó seriamente sobre las bases gnoseológicas de la teoría de sistemas que empezó a asentarse en los años setenta, tras los trabajos de L. von Bertalanffy.

22 Polo, L. (2016b), pp. 162-164. La inducción (epagogé) como captación del universal a partir de los casos singulares se encuentra en Aristóteles, Analíticos posteriores, 2, 19, 99ª $-100^{\mathrm{a}}$.

23 Cfr. Polo, L. (2016b), pp. 34-36: Polo considera correcta la propuesta aristotélica sobre la libertad y los medios, aunque limitada. Esta concepción de la economía supone que sus actividades están subordinadas a los niveles superiores de la perfección humana, es decir, el saber y la virtud.

24 Polo, L. (2016b), p. 38. 
tendencias innatas más elevadas. Se trata de la coordinación de nuestros deseos para alcanzar bienes o situaciones más o menos lejanos, y de nuestra respuesta emotiva cuando nos planteamos si estamos en condiciones de conseguirlos: hay una cierta alegría en sentirse capaz de conseguir algo, y una cierta tristeza en sentirse incapaz de ello. La afectividad animal tiene una dinámica semejante cuando anticipa los deleites gracias a una rica percepción de los fenómenos, a su memoria y a su capacidad de previsión ${ }^{25}$. El animal no se pone en movimiento si estima que no puede alcanzar el objeto de su deseo. La bestia evalúa la propia situación con respecto a lo deseado, y el resultado de la valoración, cuando es positivo, promueve la puesta en marcha en pos de aquello que parece accesible.

Por lo que respecta al animal racional, Aristóteles afirma que:

"es deleitable la actividad del presente, la esperanza en el futuro y la memoria del pasado; pero es más placentero todavía lo que depende de nuestra actividad, y por eso es más amable"26.

La armonización de los deseos con la actividad presente y los planes de futuro cambia dependiendo de la edad: en los jóvenes la esperanza surge fácilmente, mientras que en los ancianos se manifiesta avaramente, y esto está asociado a la mayor o menor experiencia de la vida. Es decir, los jóvenes se entusiasman con facilidad porque su escaso contacto con los obstáculos condiciona la ponderación. En los ancianos, precisamente la abundancia de frustraciones vividas los hace más desconfiados ante el futuro y sus propias capacidades ${ }^{27}$.

Tomás de Aquino retoma las observaciones de Aristóteles en el minucioso tratado El hombre, de la Suma de Teología ${ }^{28}$ para explicar el surgimiento de las distintas pasiones fundamentales ${ }^{29}$. En este ámbito, el pensador medieval

25 Cfr. Aristóteles, Ética nicomaquea 3, 1118 17-22.

26 Aristóteles, Ética nicomaquea 9, 1168 13-15. Las cursivas son añadidas. Sobre la alegría al sentirse capaz de algo y su relación con la esperanza, cfr. Peterson, J. B. (2018), pp. 108-109.

27 Cfr. Aristóteles, Retórica 1398 13-28; Ética nicomaquea 3, 1117ª 13ss.

28 La Suma, es decir, el compendio de la ciencia teológica del Aquinate, está compuesto por tres Partes y un Suplemento a la tercera de ellas. La Primera Parte está dividida en otras dos, y el tratado El hombre (De Homine), en el sentido del ser humano, es la Primera de ellas. De ahí la referencia tradicional a la Prima Secundae, abreviada normalmente I-II. Las partes se subdividen en cuestiones, y ésas a su vez en artículos, cuya parte central es denominada cuerpo.

29 Aquino, T. (1989), pp. 327-334, I-II, cuestión 40. Los casos de la esperanza del inconsciente (del niño y del borracho) aparecen en el cuerpo del artículo 6 de la cuestión (p. 332). El tratamiento de la esperanza como virtud teologal se encuentra en un contexto completamente distinto, en II-II, cuestiones 17 y 18. 
destaca los cuatro elementos que originan el movimiento afectivo de la esperanza: la (a) percepción de un bien (b) arduo (c) asequible (d) en el futuro. Así la distingue del simple deseo y del bien meramente deleitable ${ }^{30}$, y se comprende el lugar destacado que tiene en el conjunto de las pasiones fundamentales.

El esquema de las pasiones del Aquinate desglosa las apretadas exposiciones aristotélicas y explica el surgimiento de once sentimientos básicos. El hecho de que algunas cosas o situaciones nos atraigan y otras nos resulten desagradables, lleva a suponer que hay dos pasiones fundamentales, es decir, el amor y el odio (o rechazo en general) (pasiones 1 y 2).

$\mathrm{El}$ amor se traduce en deseo ante un bien sensible futuro (3), y en adversión cuando se trata de algo captado como nocivo (4). Cuando entra en juego el bien futuro que supone esfuerzo se encuentran la esperanza (5) o la desesperación (6). Con respecto al mal surgen el temor o la audacia (7 y 8) mientras éste no se ha presentado; una vez que se presenta, puede surgir la ira (9), que sirve para enfrentar la situación negativa. Por último, el bien deleitable presente produce alegría, mientras que el mal presente produce tristeza $(10 \mathrm{y} 11)^{31}$.

Hay una cierta subordinación en estas pasiones que realza el papel de la esperanza. Esta pasión alimenta tanto a la audacia como a la ira, motores emotivos indispensables para ponerse en marcha ante las adversidades. La esperanza se puede definir como una expectación que alimenta la proactividad.

Este minucioso desarrollo de la dinámica de las pasiones tiene sentido como principio del proceso de asimilación de informaciones (externas e internas). Sobre esta primera valoración las facultades superiores pueden elaborar sucesivas evaluaciones y conducir la toma de decisiones. La afectividad no ve más allá de lo inmediato. Este sentir y sentirse de la persona en una situación determinada, debe pasar a valorar los porqués y los cómos para aprovechar o superar esa situación. Ahí, en la deliberación, se fraguan las elecciones, las decisiones y los proyectos, y las puestas en marcha, teniendo en cuenta las experien-

30 Aquino, T. (1989), pp. 327-328, I-II, cuestión 40, artículo 1, cuerpo.

31 Aquino (1989), p. 240, I-II, cuestión. 25, artículo 3, cuerpo; cfr. Etica nicomaquea 2, 1105b23. Cfr. Arnold, M. B. (1960), p. 196. Magda Arnold (1903-2002) fue una psicóloga de origen moravo que se trasladó a Canadá y después a Estados Unidos. El sacerdote jesuita John Gasson (1903-1988) la introdujo al pensamiento antropológico de Tomás de Aquino, y gracias a la inclusión de los elementos cognitivos (estimativa, cogitativa) en la teoría de las emociones, abrió un filón que sigue dando frutos en la psicología contemporánea. Cfr. Cornelius, R. (2006). 
cias acumuladas ${ }^{32}$. Sobre estas bases se construyen los comportamientos, que van forjando las virtudes o van degenerando en vicios. En el proceso se puede experimentar esa gratificación a la que se refiere Aristóteles -“es más placentero todavía lo que depende de nuestra actividad"- y se advierte que lo importante y que vale la pena buscar es la actividad constructiva, no la gratificación en sí misma ${ }^{33}$.

Polo y Carlos Llano usan el término hegeliano "pasión fría" para referirse a la energía que dan las emociones a los proyectos de largo plazo, o sea, cuando están incorporadas en el feedback progresivo que no depende ya del entusiasmo inicial, sino de la disciplina y la colaboración continuadas ${ }^{34}$. Puede afirmarse que la esperanza es una de estas pasiones que se incorporan al ritmo cotidiano y ayudan a propulsar las elecciones de la recta razón. En este sentido cabe también referirse a la esperanza como un "depósito energético". El resto de este escrito presenta distintas dimensiones del enriquecimiento de este acumulador de recursos vitales ${ }^{35}$.

\section{COMUNICACIÓN Y DIRECCIÓN DE PERSONAS}

En el libro en coautoría Antropología de la acción directiva, Carlos Llano y Leonardo Polo explican la dirección como una conjunción de voluntades en vista de un objetivo común. Esto supone que la voluntad de quien dirige sea compartible, porque si las otras personas involucradas no quieren, todo es más

32 Sobre la importancia de la deliberación, véase Polo, L., y Llano, C. (2019), pp. 425-464, c. "VIII La intervención de la deliberación como ideal organizativo", que presenta una explicación importante sobre la comunicación en las estructuras organizativas. Acerca de la deliberación y la toma de decisiones (análisis pausado, ejecución presta), también en situaciones nuevas o de emergencia, cfr. la misma obra, pp. 356-357; 371-373.

33 Cfr. Seligman, M. P. (2003), pp. 8; 116-117.

34 Cfr. Kristjánsson, K. (2018). Kristjánsson propone una extensión del influjo de las virtudes a la dimensión afectiva para recuperar el sentido positivo de algunas pasiones que en el mundo clásico son tratadas con ambigüedad, como la compasión. El estudioso islandés no profundiza en las implicaciones antropológicas de su propuesta, como por ejemplo la idea del feedback desarrollada por Polo y Llano. Abre sin embargo una puerta para reinsertar el discurso de la recta razón en la guía de las pasiones, para desarrollar virtudes, que a su vez repercuten en el modo de percibir las situaciones propias y ajenas. Su modo de retomar la forma mentis del Estagirita es digna de atención.

35 Incluso sin atender a todas las implicaciones de la esperanza en el contexto de la antropología trascendental de Polo, se puede entender la atrevida afirmación del filósofo, que define a esta pasión como "la tendencia elevada a virtud". Polo explicó en distintas ocasiones su visión de la esperanza. El tratamiento más largo y sistemático es "El esperar", en Polo, L. (2019), pp. 238-255. El texto que ofrece una conexión más profunda con su propuesta antropológica es el capítulo "La libertad como no desfuturización del futuro y como desubicación”, en Polo, L. (2017), pp. 158-171. 
difícil, y de los seres humanos solamente se obtiene lo que están dispuestos a dar ${ }^{36}$.

Proponer una idea al grupo de trabajo está por encima del mero suscitar pasiones u ofrecer estímulos extrínsecos. Esto supone la persuasión, llegar a mover la voluntad de otros al presentarles bien algo bueno. Esta labor es marcadamente más fina que amaestrar animales o mandar a esclavos, y también más estimulante: "mandar a esclavos carece de interés", sentenciaba Aristóte$\operatorname{les}^{37}$.

La propuesta de los OKR, explicada en el parágrafo XI de este trabajo, muestra la importancia y la dificultad de elaborar y comunicar objetivos claros. Las estructuras requieren autopistas internas de comunicación para canalizar los esfuerzos de seres inteligentes y estructurados para donar.

\section{VERDAD Y VERDAD PRÁCTICA}

Polo recoge la idea clásica de la verdad como adecuación a la realidad para ponerla en relación con el trabajo estructurado. En ese sentido, compartir programas y objetivos no puede hacerse de espaldas a las condiciones reales del equipo y su entorno, pues como señala Polo, "la verdad no tiene sustituto útil” ${ }^{38}$. A renglón seguido conecta esa aseveración en términos de compromiso y esperanza, ya que sin verdad no hay orientación válida, y con la verdad se pueden vislumbrar caminos nuevos:

"En virtud de su conexión con la verdad, la esperanza forma parte de la libertad. La esperanza es tanto antecedente como consecuente. La libertad se vive en esperanza. Uno espera ser más libre, y al ser libre espera más: más verdad"39.

Polo critica severamente las propuestas ideológicas en las que se presenta al ser humano como "hecho para recibir", más que para activar sus energías y donar ${ }^{40}$. En realidad, estamos constituidos más para el trabajo que para el pla$\operatorname{cer}^{41}$, y eso significa que estamos descompensados o volcados hacia fuera, no di-

\footnotetext{
36 Cfr. Polo, L. y Llano, C. (2019), p. 406.

37 Cfr. Polo, L. (2019), p. 354.

38 Polo, L. (2019), p. 212. También Polo, L. (2016a), p. 209; Polo, L. (2015b), p. 238, "La posesión inmanente".

39 Polo, L. (2016a), p. 209.

40 Cfr. Polo, L. (2019), p. 322.

41 Cfr. Polo, L. (2019), p. 352.
} 
señados para mantener un equilibrio homeostático. Esto se nota incluso en la celebración de los logros, como la cosecha en las sociedades rurales: una fiesta que se establece para celebrar compartiendo un excedente ${ }^{42}$.

Tal desequilibrio y el carácter donal tienen mucho que ver con la libertad, el riesgo y el crecimiento. La iniciativa para dar requiere una maduración que no es inercial:

"el riesgo aparece en la forma de elección entre el crecimiento y los bienes inmediatamente al alcance. Por eso también es propio de la libertad jugar a largo plazo" ${ }^{43}$.

Es importante mantener las justas proporciones entre el riesgo y el desarrollo de la persona en el largo plazo, para no "degradar el futuro" 4 . Por eso el encuentro con la realidad se puede ver como la aceptación de un encargo, es decir, la realidad como invitación ${ }^{45}$. Los proyectos atractivos son realistas ${ }^{46}$ -de esa realidad que nos encarga algo- y convocantes: "convocar es tanto como declarar que uno solo no puede, o que el triunfo aislado no es lo que le importa" ${ }^{47}$.

Se comparte un plan y así se convierte en tarea común, y la propensión natural a donar -la efusividad- se encauza como colaboración ${ }^{48}$. Eso supone que el ser humano está hecho para co-crear, y que esa participación en la labor generadora implica ayudar a crecer a los demás. Cada uno crece en dependencia con los demás. El optimista-realista cuenta con dos posibilidades porque sabe que puede mejorar -o empeorar-. La esperanza implica tanto la insatisfacción por la situación actual ${ }^{49}$ como la conciencia de que se puede mejorar con los propios esfuerzos ${ }^{50}$.

42 Cfr. Polo, L. (2019), p. 324.

43 Polo, L. (2019), p. 325.

44 Polo, L. (2019), p. 312.

45 Cfr. Polo, L. (2016a), pp. 208-211, "El encuentro con la verdad". La verdad encarga la tarea y la inteligencia pone por obra el encargo de articular el vivir de acuerdo con este descubrimiento. Polo desarrolla este discurso incluyendo a la realidad como algo creado e inacabado. Su Hacedor lo ha querido así. Las evidencias sobre nuestra capacidad de iniciativa embonan bien con esta perspectiva en la cual participamos en la Creación llevándola adelante. Cfr. también Polo, L. (2015a), p. 27.

46 Cfr. Polo, L. (2019), pp. 371-385. La discusión sobre el error se abre con una definición de la objetividad.

47 Polo, L. (2019), p. 244.

48 Cfr. Polo, L. (1998), pp. 157-158; 163.

49 Cfr. Polo, L. (2019), p. 240 "La insatisfacción es inquietud, más no intranquilidad ni agitación. [...] Ir por más, en tanto que dar, es superior a tener. [...]. Ir por más no es tampoco salir huyendo [...] no es la fuga de un mundo inhóspito en el que no se puede vivir. La esperanza es insatisfecha no porque la situación sea mala, sino que se quiere algo mejor".

50 Cfr. Polo, L. (1998), p. 158. Véase también el capítulo conclusivo de Polo, L. (2016a).

51 Polo, L. (2016a), p. 209. 
La realidad le puede salir al encuentro "de un modo radiante", y entonces, si la persona "tiene suficiente agudeza, si no es un animal, se dice: para mí eso es imprescindible, embarco mi ser en ello. Eso es ser libre destinándose" ${ }^{51}$.

Esa especie de encantamiento necesita un campo de acción determinado. Por eso es importante que los ambientes de trabajo propicien un desarrollo compartido donde puedan realizarse esos retos y germinar nuevas iluminaciones. Se trata de apostar por la imaginación y la iniciativa de todos los implicados en la empresa:

"La creatividad del trabajador, negada radicalmente por el taylorismo, emerge en estas nuevas condiciones y ello procura notables ventajas competitivas. El pequeño invento es aprovechable. La pequeña ocurrencia del que está a pie de obra y conoce bien su trabajo, es difícil que pase por la cabeza de un ingeniero" ${ }^{52}$.

Vale la pena repetir la afirmación aristotélica "es más placentero todavía lo que depende de nuestra actividad". Tanto Polo como Doerr coinciden en la superioridad de la gratificación que da el sentirse capaces, -creciendo cada vez más en capacidad-, sobre una mención honorífica o un aumento de sueldo $^{53}$. Polo pone en relación este progreso de la tarea esperanzada con la alegría y con una lectura positiva de su crítica a la aproximación de la actividad humana como juego de suma cero. La tarea en común puede ser "un juego que no agobia: un juego alegre al que cabe apostar porque todos ganan. El último elemento de la esperanza es la alegría" ${ }^{54}$.

Es importante tener esto en cuenta para evitar que la programación de objetivos derive en un engranaje mortificante y, a la larga, insostenible.

\section{EFUSIVIDAD Y CONCRECIÓN DEL FUTURO}

Polo afrontó en distintas ocasiones la cuestión del mando y el liderazgo, poniéndola frecuentemente en el contexto del crecimiento de las personas ${ }^{55}$. En este sentido, están siempre presentes algunas de las nociones citadas más

52 Polo, L. y Llano, C. (2019), p. 410.

53 Cfr. Doerr, J. (2018), pp. 10, 15, 125, 164, 186.

54 Polo, L. (1998), p. 162. Sobre los elementos o factores de la esperanza, véase también Polo, L. (2019), pp. 251-252.

55 Cfr. Polo, L. y Llano, C. (2019), pp. 405-423, c. VIII, “¿Qué es dirigir?”. En esta línea son valiosos también otros pasajes ya citados: Polo, L. (2019), pp. 376-421, cc. VIII, IX y X, "Hacia un mundo más humano", "El mando" y "La acción de gobierno". 
arriba, como el carácter efusivo de los seres humanos, su carácter relacional, y la conexión entre la situación interna -emotividad- y la responsabilidad que se contrae con el entorno social.

Una cuestión importante y muy debatida desde que Peter Drucker la puso en primer plano en los ambientes de la dirección de empresas a partir de los años cincuenta, es la determinación de los objetivos ${ }^{56}$. Polo plantea la cuestión a nivel antropológico explicando que la racionalidad hace capaces a los seres humanos de estar por encima del flujo temporal y dirigir de este modo su actividad. El ratchet effect se manifiesta en esta posibilidad de pararse a pensar para dirigir las acciones y hacer planes ${ }^{57}$. Es una de las manifestaciones de la biperteleologización, es decir, la capacidad de conducir hacia planos distintos e inéditos, realidades ya marcadas por un orden natural. Ahí se pueden encuadrar la libertad de elección y la libertad de crecimiento $^{58}$.

Como ya se ha recordado, la inteligencia ve más allá de los sentimientos, y además puede ser cultivada para entender más y tomar mejores decisiones, y ver con mayor agudeza los distintos niveles de coordinación de esfuerzos. También se puede planear arriesgando con generosidad en objetivos de largo plazo y de calidad: "dime lo que esperas y te diré cómo vives; uno vive según lo que espera" ${ }^{59}$.

Otra idea que atraviesa el planteamiento del mando en Polo es el carácter no desfuturizable del futuro, o sea, la conciencia de que podemos anticiparlo intelectual e imaginativamente, y que su realización depende de lo que hagamos. Podemos -y debemos- intervenir para generar escenarios que, obviamente, todavía no son reales ${ }^{60}$. Hay otros descubrimientos de la psicología contemporánea que se ajustan a esta elaboración poliana, como se verá en el apartado siguiente.

56 Cfr. Greenwood, R. (1981), Doerr, J. (2018), pp. 23-26.

57 Véase el parágrafo IV, supra.

58 Cfr. Polo, L. (2019), pp. 371; 378-379.

59 Polo, L. (2019), pp. 272-273. Para Drucker no era indiferente la calidad de los objetivos y su compatibilidad con el crecimiento de la persona. Covey, S. R., Merrill, R. y Merrill, R. (1994) subrayan más decididamente la necesidad de que los objetivos personales, basados en altos ideales, sean el faro de la propia vida, para generar planes de largo plazo basados en una disciplina personal medible también en el corto plazo.

60 Cfr. Polo, L. y Llano, C. (2019), p. 456. Cfr. Polo, L. (2018), p. 289, y Polo, L. (2019), p. 402. En la nota 35 se han citado textos que asocian esta noción con la esperanza. Sobre la importancia del pensamiento orientado hacia el futuro en la dirección de empresas, véase Johnson, M. y J. Suskewicz, J. (2020). 


\section{PLANEACIÓN VS ENSOÑACIÓN}

Baumeister asocia la peculiaridad de la actividad humana a cómo manejamos nuestro futuro. Este psicólogo social y otros autores se han inspirado en la visión de William James sobre la autonomía y el comportamiento finalizado o intencional (purposive behavior) ${ }^{61}$.

La base de esos estudios es la comparación de distintos procesos mentales, que van desde la divagación y el soñar con los ojos abiertos, a la toma de decisiones y a la planificación de programas concretos. Con esa base tratan de explicar la dinámica de la deliberación y la elección, que son fundamentales para ordenar la secuencia de los eventos futuros y requieren un esfuerzo de concentración particular.

Para establecer un plan hace falta una cierta energía y la presteza ante los desafíos. Los planes y los acuerdos no "ocurren", sino que derivan de un esfuerzo de focalización en el que el individuo sopesa su compromiso. La planeación tiene que ver con lo que cada uno desea porque le da un significado, el cual se basa en motivaciones de distintos tipos. Se trata de un pasaje crucial en la administración del tiempo moderando nuestros deseos. Baumeister explica de esta manera sus reflexiones sobre los resultados de una amplia encuesta sobre el peso de la planificación en las deliberaciones de los sujetos entrevistados:

"una de las razones fundamentales que dan cuenta de la importancia de la planeación es que ésta conecta los acontecimientos a través del tiempo, lo cual es de suyo un enlace significativo. Al mismo tiempo, enriquece la red asociativa de cualquier pensamiento o idea [...] [hay] un patrón general que enlaza la plenitud de significado en el tiempo".

Y concluye afirmando que "los pensamientos que obtenían puntajes más altos eran los que combinaban el pasado, el presente y el futuro"62.

El esfuerzo para entrelazar armónicamente los distintos capítulos de la propia vida tiene efectos gratificantes en el ánimo, que se ve permeado de serenidad, relaja del estrés y aminora o elimina la irritación y la inquietud.

En otro de sus trabajos, Baumeister y sus colegas presentan de nuevo esta teoría de la prospección pragmática (pragmatic prospection). También ahí subra-

61 He presentado algunas de estas nociones en Mercado, J. A. (2021), pp. 150-154.

62 Baumeister, R. F. (2016), pp. 168-169. 
yan la peculiaridad de los seres humanos como configuradores de su futuro ${ }^{63}$. Los autores definen la prospección pragmática como la capacidad "de pensar acerca del futuro como guía de las acciones en vistas a la obtención de resultados deseables" ${ }^{4}$. Esta formulación coincide con los principios aristotélicos: los seres humanos pueden pensar en su futuro de manera que pueden modelarlo. Esto supone la capacidad de evaluar la factibilidad del proyecto y la distinción entre deliberar y ejecutar, entre planear y predecir ${ }^{65}$. El modo como Aristóteles presenta los efectos anímicos de la conexión significativa entre los eventos citada anteriormente, así como la conciencia de poder intervenir en ellos, también conecta con estos descubrimientos recientes: abordar seriamente la programación de las actividades fomenta la serenidad, lo cual a su vez ayuda a concentrarse mejor en la actividad. Todo esto supone asimismo un uso disciplinado de la imaginación, que prevé y combina situaciones distintas en estrecha colaboración con la inteligencia.

Estructurar las ideas o proposiciones generales a través de planes abordables significa transformarlas en un relato personal. Esta narración ${ }^{66}$ implica un monitoreo permanente tanto del programa trazado como de la evolución de las propias facetas emocionales a lo largo del recorrido ${ }^{67}$. Comparar de este modo la propia afectividad lleva consigo un reconocimiento de la capacidad evaluadora de las emociones, es decir, que éstas nos dan continuamente información sobre nuestra situación interna. Como se verá en la explicación del sistema basado en los resultados clave (OKR), esta faceta de revisión y mejoramiento se puede llevar a cabo sistemáticamente en los grupos de trabajo y en las organizaciones.

Volviendo sobre las conclusiones de Baumeister, Oettingen y Vohs, vale la pena destacar que el término narrativa implica que conducirse desde el presente hacia "un resultado futuro deseado requiere una concatenación de acciones y eventos significativa y con frecuencia causalmente interrelacionados" ${ }^{8}$. Además, concluyen que "los planes vinculados a los valores más altos de las

63 Cfr. Baumeister, R. F., Oettingen, G. y Vohs, K. D. (2016), pp. 8, 13.

64 Baumeister, R. F., Oettingen, G. y Vohs, K. D. (2016), p. 3.

65 Cfr. Baumeister, R. F., Oettingen, G. y Vohs, K. D. (2016), pp. 3-5.

66 Cfr. Baumeister, R. F., Oettingen, G. y Vohs, K. D. (2016), pp. 7-8; 13.

67 Cfr. Baumeister, R. F., Oettingen, G. y Vohs, K. D. (2016), pp. 8-11.

68 Baumeister, R. F., Oettingen, G. y Vohs, K. D. (2016), p. 8. Véase Aristóteles, Ética Nicomaquea, 1176 a155 ss. 
personas suelen dar mejores resultados que otros" ${ }^{9}$. Los estudiosos comentan que conectar el futuro con el presente basados en narraciones plenas de sentido "puede mejorar nuestra toma de decisiones y los resultados a largo plazo"70.

Estas conclusiones sobre el bienestar psicológico que deriva de la programación significativa coinciden con el tratamiento aristotélico de la esperanza y su afinación por parte de Tomás de Aquino y los desarrollos de Polo. Es una gratificación que sostiene en distintas etapas del proyecto, y en ese sentido ayuda a la perfección de la obra, como los placeres en general.

También conviene señalar que cuando a ese esfuerzo sigue una puesta en marcha ordenada y verificable, el feedback emotivo suele seguir nutriendo positivamente a la actividad, normalmente sin sobresaltos ${ }^{71}$. Es una fuente de gratificación sin exaltación, que tiene correlaciones profundas con los estados de ánimo donde se ven las soluciones con mayor claridad ${ }^{72}$. Aunque pueda parecer una afirmación insustancial, algunos psicólogos aprovechan análisis recientes sobre la expresión de La Gioconda, de Leonardo da Vinci, para explicar que una alegría sin estridencias es la situación más normal -y también frecuente en muchos lugares-, y que gracias a ella se está en condiciones, por ejemplo, de conectar mejor los medios para encontrar soluciones ${ }^{73}$.

\section{MANDO Y SISTEMA}

Vale la pena mencionar algunas observaciones de Polo con las que se puede concretar la gestión de la descompensación humana en las organizaciones, para conectarlo después con una propuesta práctica de coordinación del trabajo. El filósofo insiste, por ejemplo, en la importancia de la claridad de la información, tanto sobre los fines como sobre los medios ${ }^{74}$; la importancia de verificar los resultados y la presencia constante y no perturbadora del direc-

69 Baumeister, R. F., Oettingen, G. y Vohs, K. D. (2016), p. 10. Los autores no plantean una discusión sobre la objetividad de lo que las personas valoran más o menos, aunque por el contexto resulta evidente que se trata de tener relaciones sociales y familiares estables; planes de progreso en el propio trabajo, etc.

70 Baumeister, R. F., Oettingen, G. y Vohs, K. D. (2016), p. 8.

71 Cfr. Diener, E. y Biswas-Diener, R., (2008), pp. 165-180.

72 Cfr. Fredrickson, B. (2013) y Fredrickson, B. (2018). Fredrickson ha trabajado intensamente en la clarificación de las correlaciones entre de la resonancia afectiva, sus bases neurobiológicas, las actitudes y las relaciones constructivas, y sus consecuencias en la puesta por obra de los proyectos.

73 Cfr. Diener, E. y Biswas-Diener, R., (2008), pp. 219-220.

74 Cfr. Polo, L. y Llano, C. (2019), p. 406. 
tivo; el monitoreo de los suministros y la distribución de la carga de trabajo; aplicar la capacidad de síntesis en todas esas facetas de su trabajo ${ }^{75}$. Un resultado normal de una gestión bien llevada es el aumento de la confianza ${ }^{76}$. También da un lugar relevante a la búsqueda de colaboradores valiosos y a la búsqueda de reemplazos para el gobierno ${ }^{77}$.

Aunque Polo acoge la observación aristotélica sobre la prudencia del buen general, quien "se sirve del ejército de que dispone haciéndole rendir todas sus posibilidades en el combate" 78 , su idea de la persona que gobierna no se concentra en la catalogación de las cualidades personales. Polo subraya especialmente la participación en el gobierno y la toma de decisiones: "no conviene tomar decisiones en solitario", y lo asocia a una concepción del liderazgo ajena a la excesiva personalización:

"El líder es un sistema; es el liderazgo. El líder nunca es una persona. Nadie es suficiente él solo para dirigir de acuerdo con la virtud de la fortaleza y de acuerdo con la virtud de la templanza, pues eso no es posible"79.

Me parece que esta concepción del liderazgo es complementaria a la que expone Henry Mintzberg en Simply management. Mintzberg se opone garbosamente a la oposición entre leader y manager, y presenta numerosos casos en los que se muestra que quien manda no puede coordinar el trabajo como si estuviera en un helicóptero, impartiendo órdenes sin tener un contacto real con las situaciones, sin sentir el pulso de las circunstancias ${ }^{80}$.

La relación entre el líder y las condiciones de trabajo son tan variadas como las organizaciones, pero el liderazgo es siempre un marco de actividades para potenciar la iniciativa de los participantes. El liderazgo no es el líder, sino un ámbito estructurado de colaboración, un plexo organizativo en el cual todos los miembros de la institución "actúan mejor que en cualquier otra"

Si se comparten objetivos altos y se promueve la iniciativa, se evita la rutina y crece la motivación de manera natural. En cambio, "el que pertenece a

75 Cfr Polo, L. y Llano, C. (2019), pp. 442, 447.

76 Cfr. Polo, L. (2018), pp. 288-290, y Polo, L. (2019), pp. 379-380.

77 "Un directivo que es incapaz de rodearse de buenos colaboradores, que le puedan sustituir, no está jugando bien [...] En esa tontería incurrimos los mortales con mucha frecuencia". Polo, L. y Llano, C. (2019), p. 368.

78 Aristóteles, Ética nicomaquea, 1101ª3-6.

79 Polo, L. (2019), p. 418.

80 Cfr. Mintzberg, H. (2013).

81 Polo, L. (2019), p. 380. Cfr. Castillo, G. (2017). 
una organización renqueante se desanima" ${ }^{\prime 2}$. La natural efusividad humana exige que a la persona se le ponga en condiciones de dar más ${ }^{83}$; la persona medianamente inteligente y valiosa no debe ser degradada con un tratamiento de autómatas ${ }^{84}$. Todo esto se conecta con la gratificación normal, moderada y permanente a la que se ha hecho referencia en los parágrafos anteriores ${ }^{85}$.

\section{EL SISTEMA OKR}

\section{Qué son los OKR}

El sistema de trabajo basado en objetivos + resultados clave (objectives, key results), y una medición continua es una creación de Andy Grove, madurada en la cultura de Intel en los años setenta, construida en parte sobre la ya mencionada gestión por objetivos, de Drucker. John Doerr explica con detenimiento esta estrategia en Measure what matters.

El sistema también tiene afinidades con algunas ideas centrales de First Things First, de Covey, y las distintas derivaciones en las propuestas "Get things done" (GTD) ${ }^{86}$. El marco de Covey abarca las distintas dimensiones de la actuación personal y la autoevaluación, mientras las estrategias GTD se enfocan más a la eficacia, sobre todo en la gestión individual del tiempo. Las tesis de Grove-Doerr se enfocan al trabajo en las organizaciones y, aunque se centran en los resultados, suponen un modo de ver y gestionar el tiempo humano que admite una lectura desde las ideas de Polo que he mencionado hasta ahora.

Doerr describe la perspectiva de su propuesta con el aforismo "las ideas son fáciles. La ejecución lo es todo" ${ }^{87}$. Con esto quiere decir que la habilidad para llevar a cabo los planes - "el papel lo aguanta todo"- vale mucho más que la gestación de los mismos. También implica, como se irá viendo, que esa ha-

82 Polo, L. (2019), p. 379.

83 Cfr. Polo, L. (2019), p. 381.

84 Cfr. Polo, L. y Llano, C. (2019), pp. 405-407, 412, 420.

85 También cabría asociar estas nociones a la ideas del compromiso (engagement) y la inspiración como resultados y causas de una buena gestión y una mayor productividad y eficiencia, de Drucker, quien sentenciaba "quienes llevan a cabo las cosas con perfección aman lo que hacen". Cfr. Bergstrand, J. (2013).

86 Covey, S. R. y Collins, J. (2013), y Allen, D. (2015).

87 Doerr, J, (2018), c. 1. 
bilidad puede mejorar gracias a la misma metodología de trabajo, por el feedback continuo de un proceso recurrente. Doerr está convencido de que el método OKR puede transformar el modo de trabajar y es un soporte magnífico para llegar donde hace falta. A su modo, asume la idea de que la razón práctica es razón recta y co-rrecta, como insistía Fernando Inciarte ${ }^{88}$.

La metodología de los OKR y su medición ayuda a delimitar las cosas que hay que hacer y cómo llevarlas a cabo correctamente, según el ideario de la empresa y los objetivos de mediano y largo plazo. En esto coincide con los puntos de referencia significativos, según los describen Baumeister y sus colegas, como se ha visto en el parágrafo IX. De este modo se canalizan y coordinan los esfuerzos de todos los participantes para obtener los resultados adecuados (the right goals).

Doerr reconoce que su propuesta metodológica no puede sustituir a la sensatez, a un liderazgo firme y a una cultura innovadora en la institución. Más bien, cuenta con todo ello y facilita su sano desarrollo a través de realizaciones bien calibradas al principio y revisadas en periodos delimitados de tiempo ${ }^{89}$.

Suponiendo que el personal de la organización está alineado con la misión institucional, la dirección debe plantear objetivos claros. Después viene la distribución del trabajo, es decir, que cada equipo y/o cada persona sepa lo que le corresponde en ese plan, y que todos sepan cómo evaluarlo y en qué lapso de tiempo. Además, tanto los objetivos como las mediciones son públicas y se actualizan con la misma frecuencia que se llevan a cabo las evaluaciones.

Doerr subraya una verdad evidente: un objetivo es, ni más ni menos, lo que hay que conseguir. Sin embargo, frecuentemente la distancia que media entre un ideal de la misión, y los objetivos para el próximo trimestre, son poco claros, o francamente equivocados ${ }^{90}$.

Como en un tratamiento médico, se da una conexión profunda entre las dosis de la medicación y la supervisión de los resultados, a través de los cuales se puede revisar la dosificación siguiente. Si esto no se hace con atención, fácilmente surgen problemas sistémicos en la organización debidos a la estrechez de miras, a comportamientos inmorales, a la asunción desmedida de riesgos, que se manifiesta en la mengua de la colaboración y de la motivación.

88 Cfr. Mercado, J. A. (2021), pp. 136-137 y Polo, L. y Llano, C. (2019), p. 419.

89 Cfr. Doerr, J, (2018), p. 6.

90 Cfr. Doerr, J. (2018), c. 1, pp. 7-9. Doerr cita casos claros de fines torcidos, como los de Enron o como la desastrosa escalada al Everest de 1996, durante la cual murieron ocho excursionistas. 
Cuando los individuos tienen prioridades contradictorias, sin sentido, o una asignación fluctuante o arbitraria de objetivos, pululan la frustración, el cinismo y el descorazonamiento ${ }^{91}$. En cambio, la realización de los fines y su reconocimiento promueve el involucramiento de todos trabajadores. Más que medir "cosas", se busca cómo evaluar tangiblemente los logros, y las motivaciones extrínsecas sirven simplemente como un resello de algo que ya se sabe. Los OKR, afirma Doerr, se refieren a algo más profundo, es decir, "al valor intrínseco del trabajo en sí mismo" $"$.

Además del enfoque y la claridad, los objetivos deben contener el justo nivel de desafío según las circunstancias, es decir, deben ser asequibles. En esto la conexión con la esperanza de los clásicos es neta: la esperanza surge cuando el objeto conveniente se ve como algo alcanzable, aunque arduo. También es fácil conectar con el discurso de Baumeister y sus colegas, sobre el esfuerzo que supone plantearse fines específicos y demarcados temporalmente.

Doerr enfatiza la importancia de la satisfacción por la obtención de resultados, sin prestar atención a lo que sí indica Baumeister sobre la gratificación resultante de una buena formulación de planes. Me interesa subrayar la emergencia de las sensaciones positivas en las distintas etapas de los procesos, pues forman parte de esa relativa felicidad psicológica a la que he hecho referencia al mencionar el trabajo de Diener y Fredrickson, en las líneas finales del apartado IX.

\section{La calidad de los objetivos}

Se ha hecho una breve referencia a la importancia de plantearse objetivos altos y nobles, y a la importancia de definir algunos de ellos detalladamente, en el contexto del pensamiento de Polo. Para considerar la bondad de los objetivos en el planteamiento de Doerr, hay que suponer que las empresas comerciales se fijan ideales y objetivos generales constructivos, pero no se plantean cuestiones sobre sus bases morales. En ese sentido, el sistema de los OKR es perfectamente compatible con exigencias antropológicas profundas. Donde es

\footnotetext{
91 Cfr. Doerr, J. (2018), c. 1. La falta de objetivos hace precaria cuando no imposible la programación; sin resultados que alimenten nuevos proyectos, suele sobrevenir el aburrimiento. Cfr. Polo, L. (2019b), pp. 265-285, c. VI. "Educar el interés". Véase también el texto citado en la nota 82. Un estudio de esta dinámica psicológica, basada sobre el esquema de las pasiones de Tomás de Aquino, se encuentra en Arnold, M. B. (1960), p. 190.

92 Doerr, J. (2018), c. 10.
} 
claramente más fino es en lo que podríamos llamar la parte artesanal de los objetivos y los resultados clave.

Los resultados clave deben ser pocos e indicar puntos concretos de mejora. Una buena parte de ellos puede provenir de la dirección, pero a partir de estos, los equipos de trabajo deben fijar sus propios sub-objetivos. Doerr propone un ejemplo para explicar los distintos niveles de consistencia de una meta, con un ejemplo automovilístico: ante el objetivo "ganar la próxima carrera", se pueden plantear resultados clave de manera más o menos eficaz ${ }^{93}$ :

Tabla 1. Objetivo general: ganar la próxima carrera

\begin{tabular}{l|l|l|l} 
Calidad & Baja & Media & Alta \\
\hline RC1 & $\begin{array}{l}\text { Aumentar la velocidad } \\
\text { por vuelta }\end{array}$ & $\begin{array}{l}\text { Aumentar la velocidad } \\
\text { por vuelta en un 2\% }\end{array}$ & $\begin{array}{l}\text { Aumentar la velocidad } \\
\text { por vuelta en un 2\% }\end{array}$ \\
\hline RC2 & Reducir el tiempo en box & $\begin{array}{l}\text { Reducir el tiempo en } \\
\text { box en 1" }\end{array}$ & $\begin{array}{l}\text { Probar en túnel } \\
\text { de viento 10 veces }\end{array}$ \\
\hline RC3 & & & $\begin{array}{l}\text { Reducir el tiempo en } \\
\text { box en 1" }\end{array}$ \\
\hline RC4 & & & $\begin{array}{l}\text { Reducir los errores en } \\
\text { box en un 50\% }\end{array}$ \\
\hline RC5 & & & $\begin{array}{l}\text { Practicar parada en box } \\
\text { una hora cada día }\end{array}$ \\
\hline
\end{tabular}

En la Tabla 1, se clasifican como resultados clave "de baja calidad" los que no son lo suficientemente específicos. Los resultados clave "de calidad media" son mejores porque son medibles y específicos, pero no llegan al detalle sobre cómo reducir el tiempo de las paradas en boxes como sí lo hacen en cambio los resultados clave más sólidos o "de alta calidad". En resumen, los resultados clave deben estar bien delimitados, ser específicos, circunscritos en el tiempo, medibles, y garantizar el control de calidad. En el caso del equipo de automovilismo, los resultados clave combinan elementos cuantitativos y cualitativos, así como resultados de calidad que ayudan a evitar atajos contraproducentes $^{94}$.

93 Cfr. Doerr, J. (2018), c. 4, tabla 4.2.

94 Cfr. Doerr, J. (2018), c. 4 Los ejemplos del autor muestran, entre otras cosas, que establecer los objetivos no es sencillo y puede requerir tiempo. La revisión frecuente ayuda en el proceso de mejora. 
Doerr sugiere que haya un "pastor" de los OKR: todos participan, y eso promueve la responsabilidad y el compromiso, pero es normal que haya resistencias y retrasos. Es útil por eso que haya alguien a cargo de mantener al resto del equipo u organización responsable de establecer y trabajar hacia sus OKRs.

Hasta ahora se ha tratado de "OKR comprometidos", o muy ligados a un objetivo inmediato. Para potenciar las capacidades del equipo se requieren también "OKR de estiramiento" (stretch OKRs). Podríamos llamarlos ignifferos, de encendimiento, pues están ligados a las aspiraciones más altas de la organización, donde hay que conjugar una exigencia que mantenga relativamente incómodo al equipo, porque los objetivos no parecen realmente al alcance de las posibilidades del momento. Se trata de tener al equipo en una situación de eu-stress, o sea, de mantener una presión gestionable, que los empuje a obtener más de lo que se creía posible ${ }^{95}$.

La revisión continua llevará a plantear nuevos resultados y a desechar los que ya no tienen sentido. En esas revisiones hay que puntuar con una escala cuánto se ha conseguido en cada renglón, y reflexionar sobre lo que ha ido mejor y lo que no ha ido tan bien. De ese modo se pueden relativizar tanto los éxitos como los fracasos, y en todo caso se afinan mejor los detalles del ciclo siguiente.

Es importante fijar los periodos de evaluación dentro del equipo, con la frecuencia que lo requiera el tipo de trabajo, de acuerdo con los objetivos generales, y contar con los instrumentos digitales adecuados: documentos compartidos, intranet, cloud, etc ${ }^{96}$.

\section{Potencialidades del sistema OKR}

Doerr desglosa los alcances o superpowers de los OKR en cuatro puntos:

1. Enfocan la atención y mantienen la adhesión a las prioridades ${ }^{97}$;

95 La conocida teoría del flow, de M. Czisztenmihalyi sostiene que la situación de flujo mental, en la que el individuo se concentra fructuosamente en una única actividad, suele darse cuando el episodio concreto presenta una dificultad ligeramente superior a la que se ha afrontado con anterioridad. De este modo la habilidad crece para salir airosa en el nuevo reto. Se trata de otro aspecto de la motivación ligada a la esperanza. Cfr. Ribera, A. y Ceja, L. (2017), p. 108.

96 Doerr cita numerosos casos de empresas con objetivos semanales, que a su vez tienen revisiones mensuales. El ciclo semanal coincide con la propuesta de First Things First, de Covey, para el rendimiento personal.

97 Cfr. Doerr, J. (2018), c. 4. 
2. Alinean la actividad de todos los participantes con los objetivos generales, pues son públicos ${ }^{98}$. Simultáneamente, promueven la conexión intra e inter grupalmente. Que los OKR sean públicos también ayuda a aprovechar los avances de los demás y a evitar repeticiones y solapamientos innecesarios.

3. Facilitan el seguimiento99: a través de la puntuación y la evaluación se renuevan los OKR útiles, se retocan los deficientes y se eliminan los obsoletos. nido $^{100}$.

4. Mantienen una cierta tensión para promover un crecimiento soste-

La tríada conversaciones-feedback-reconocimiento (CFR) son como la caja de herramientas de los $\mathrm{OKR}^{101}$ :

1. Conversaciones. En las conversaciones periódicas, se reflexiona con cada empleado sobre el desarrollo de los resultados y se buscan soluciones; se le ayuda a reenfocar su posición y a sugerirle líneas de mejora personal, y se le anima a que opine sobre la evolución del trabajo y de la empresa.

2. El feedback o retroalimentación se da en distintos momentos, y es necesaria porque no siempre es fácil distanciarse de la propia labor para valorarla en el contexto ${ }^{102}$.

3. El reconocimiento debe ser tanto privado como público, y centrado en las acciones. Es importante establecer las modalidades de este reconocimiento en cada organización.

Esta combinación de elementos se encuentra en las culturas empresariales "de alta motivación" 103 . Además, para Doerr estos instrumentos están por

\footnotetext{
98 Cfr. Doerr, J. (2018), c. 7, pp. 77-90.

99 Cfr. Doerr, J. (2018), c. 10, pp. 114-127.

100 Cfr. Doerr, J. (2018), c. 12, pp. 135-144.

101 Cfr. Doerr, J. (2018), c. 15, pp. 176-189.

102 La cuestión es relevante y requeriría un estudio aparte, pues dar y recibir retroalimentación requiere buenas disposiciones y entrenamiento. Uno de los puntos fundamentales es la adaptación de los gestores del personal para trabajar con el feedback, de manera que los trabajadores asuman el protagonismo. Es decir, la compartición de las evaluaciones debe ayudar a cada quien a hacerse cargo de sus logros y sus fallos, para afinar la mira cuanto antes. Simultáneamente se debe procurar que cada sujeto tome la iniciativa en esas conversaciones, porque obviamente la estará tomando cada vez más en el establecimiento y obtención de las metas. Cfr. Doerr, J. (2018), c. 2, p. 87; c. 9., p. 112; c. 18, pp. 216-217, 222; c. 19, pp. 226-227, 230.
}

103 Doerr, J. (2018), c. 18, pp. 216-217; p. 284. 
encima del establecimiento de los resultados. El autor sigue en este punto otra idea de Drucker para enfatizar que la tarea más importante del directivo es:

"la relación con las personas, el desarrollo de la confianza mutua, la identificación de la gente, la creación de una comunidad [...] eso no se puede medir y tampoco es fácil de definir, pero además de ser una función clave, solo [el director] puede realizarla"104.

El método, insiste Doerr, no es provechoso solamente para encarrilar y mantener el norte grandes empresas, sino para potenciar las capacidades de cualquier organización.

\section{CONCLUSIONES}

He planteado un recorrido sembrado de nociones antropológicas para conectarlas con algunos descubrimientos recientes de la psicología social y con una propuesta de organización del trabajo muy concreta, basada en los objetivos y los resultados clave (OKR). La línea del discurso asocia principios generales del comportamiento humano, como la sindéresis y la capacidad de previsión intelectual, para ver cómo se integran con ellas las emociones fundamentales, especialmente la esperanza.

Por lo que respecta a la sindéresis, la lectura que hace Polo es manifiestamente proactiva y positiva: "haz todo el bien que puedas y no te canses de hacerlo"; a pesar del cansancio, hay que seguir haciendo el bien. Para mantener esa tensión hacia el bien que se expresa en la sindéresis, hay que construir sobre la base de las facultades, y tener en cuenta las emociones positivas, que llevan a celebrar la obtención de los objetivos comunes. La efusividad humana traducida en principio operativo general -la sindéresis- está llamada a integrar la iniciativa, la organización, la alegría, la esperanza y la fiesta. Me parece que la concreción que promueve la metodología de los OKR está en sintonía con estos principios antropológicos.

El papel rector de las facultades en ese rico proyecto vital toca a la inteligencia, que evalúa las situaciones externas y el estado de ánimo, para deliberar y decidir sobre lo que conviene hacer y a qué ritmo. En las organizaciones se formaliza la gestión de estos principios: se publican horarios, se asignan car-

\footnotetext{
104 En Doerr, J. (2018), p. 176. La fuente es el testimonio de un directivo recogido en Zak, P. (2013), www.drucker.institute/thedx/measurement-myopia/ (cons. 25/01/2022).
} 
gos, funciones, protocolos, etc. de acuerdo con una misión y con objetivos de mayor o menor importancia y envergadura. Eso es importante porque se trata de la administración del tiempo humano, cuyo eventual desperdicio es un asunto grave.

Me he permitido una pequeña digresión para subrayar una coincidencia metodológica importante entre proyectos de peso en psicología contemporánea, y un aspecto de la filosofía de Polo, es decir, su idea de la inducción como aproximación sistémica y holística. Las conexiones que presenta el autocontrol con otras actividades de la mente, según las descripciones de Tomasello y Baumeister, presentan notables coincidencias con las explicaciones de rubro aristotélico de Polo sobre el cuerpo humano y las correlaciones entre sus órganos.

Las emociones pueden ayudar más o menos en la promoción de las actividades, pero es la inteligencia, con su poder persuasivo, quien debe dictar el orden: solo la inteligencia pone en perspectiva el futuro y trata de coordinarlo con los ideales de vida, deliberando para organizar los medios en vistas de un fin.

Aunque las emociones no estén capacitadas para guiar la acción, la resonancia afectiva de los esfuerzos, los logros, los fracasos, el cansancio, el aburrimiento, etc. tienen un papel relevante. Saber qué nos produce satisfacción o gratificación es de gran utilidad para aprender a cultivar y apreciar los frutos emotivos: la satisfacción de un logro que ha requerido esfuerzo tiene que ver con el placer como perfección de la acción y fomento de su desarrollo. Hay una concordancia entre hacer el bien y sentirse bien. Esa forma de alegría se da en grados distintos, dependiendo de varios factores.

En la vida diaria, las gratificaciones suelen ser "de ordinaria administración”, y deben ser relativamente abundantes para ayudar a mantener un tono emotivo que sostenga al crecimiento. Las virtudes y la "pasión fría" se construyen cada día con esfuerzos continuados, y la reatroalimentación que dan suele ser positiva, ya que ayudan a que cada vez se puedan hacer más y mejores cosas; a que se pueda mejorar la toma de decisiones y la gestión del tiempo.

La estabilidad anímica sostenida por las virtudes está asociada a una satisfacción o felicidad psicológica de vital importancia, porque trabajar siempre a contracorriente de la propia interioridad -por ejemplo, el excesivo estrés derivado de distintas circunstancias; horarios despropositados; descuido de las otras facetas de la vida, como la familia y la salud- es desgastante y, a 
mediano y largo plazo, negativo para la persona, sus colegas, su familia, y la misma empresa.

Es decir, que las gratificaciones moduladas con una actividad eficiente tienen que ver con nuestra salud física y mental. Esa alegría moderada tiene mucho que ver con la esperanza, tema sobre el cual Polo ofrece ideas muy concretas: tiene que haber una tarea exigente, en la que se está comprometida con otras personas; hay un cierto riesgo y determinados medios; normalmente hay posibilidad de pedir ayuda, y siempre hay una responsabilidad por llevar a cabo la tarea. He presentado algunas ideas de Polo sobre esta traducción de los grandes principios morales a las organizaciones: misión alta, con ideales claros, y objetivos bien definidos; información clara y eficaz; revisión frecuente de los resultados y los medios para continuar.

Un filón de la psicología social contemporánea ha conectado los estados de ánimo con la preparación de planes, es decir, al funcionamiento de la imaginación guiado por la razón en vistas a fines concretos, y basados en los principios o valores de las personas: incluso en situaciones controladas (ejercicios en equipo, entrevistas) los efectos anímicos son muy distintos a los que se registran cuando los experimentos plantean planes genéricos. La satisfacción se da incluso en ejercitaciones mentales relativamente simples: la visión prospéctica de inspiración jamesiana desarrollada por Baumeister y sus colegas, se conjunta bien con el principio más general de Polo sobre la no desfuturización del futuro: la calidad y la precisión de nuestros proyectos tiene una importancia capital.

En las empresas deben promoverse estos dinamismos a nivel grupal. Polo afirma que el líder y el liderazgo no son una persona, sino un sistema en el que se pone a los individuos en condiciones de dar más, de expresar su potencial del mejor modo posible. Sus ideas sobre los objetivos y la comunicación no van más allá. La evolución de las teorías de management basadas en los objetivos ofrece propuestas estructuradas que responden en buena medida a la necesidad de "ponerle pies" a los principios de la organización a partir de la antropología de Polo. En ese sentido, la propuesta de los objectives and key results, presenta con detalle una metodología compatible con esos principios del filósofo español.

El método presupone una misión de cada organización y los principios éticos reconocidos de la sociedad liberal contemporánea, así como un marco legal sólido. En este terreno es fundamental plantear objetivos inspiradores y objetivos más o menos inmediatos subordinados a ellos, en periodos de tiempo 
determinado y un sistema de verificación frecuente. Los objetivos, incluidos los de la dirección y las de cada equipo de trabajo, son públicos.

La medición "devlo que cuenta" es fundamental, para poder tener feedback dentro del equipo y desde las otras instancias interesadas: la dirección debe intervenir para reconocer y/o mejorar los resultados; los equipos saben a qué se dedica cada uno de los otros y a qué no se dedica (o a qué no se deberían dedicar); los resultados se comparten, las superposiciones y las interferencias se limitan, etc.

Sobre esas informaciones se ayuda a los trabajadores para estimularlos en lo que hacen bien; a darse cuenta de los aspectos de su trabajo que quizá no advierten por falta de perspectiva; a celebrar los éxitos con moderación y a reconocer los esfuerzos.

Es probable que este sistema pueda resultar excesivamente estresante y controlador, y quizá solamente aplicable a organizaciones de ambientes altamente competitivos. Me parece que adaptarlo a las exigencias de la misión institucional, a una dirección firme y al ideal de crear una cultura interna de innovación y colaboración es plausible en cualquier tipo de empresa, también en las que no tienen finalidades de lucro.

El planteamiento OKR no se contrapone a la selección de personal cualificado, y mucho menos al desarrollo de actitudes virtuosas. Es más, requiere de competencias o soft skills que basadas en una preparación exigente y profesional. Un entrenamiento adecuado en este campo puede potenciar la delicadeza y la buena voluntad para que sean instrumentos eficaces de crecimiento en una comunidad de trabajo.

Todos estos instrumentos pueden y deben funcionar en el contexto de la misión esperanzada de la que habla Polo. 


\section{BIBLIOGRAFÍA}

Allen, David (2015), Getting Things Done: The Art of Stress-Free Productivity, Penguin Publishing Group, Nueva York.

Aquino, Tomás de (1989), Suma de teología, BAC, Madrid.

Aristóteles (1984) The Complete Works, Barnes, Jonathan (ed.), Princeton University Press, Princeton.

Arnold, Magda B. (1960), Emotion and Personality, Columbia University Press, Nueva York.

Baumeister, Roy F., (2005), The Cultural Animal. Human Nature, Meaning and Social Life, Oxford University Press, Oxford.

Baumeister, Roy F. (2016), "Pragmatic Prospection", en Seligman, Martin E. P., Railton, Peter y Sripada Chandra (autores y eds.), Homo Prospectus, Oxford University Press, Oxford, pp. 157-189.

Baumeister, Roy F., Oettingen, Gabriele y Vohs, Kathleen D., (2016), "Pragmatic Prospection: How and Why People Think About the Future", Review of General Psychology, vol. 20, n. 1 (2016), pp. 3-16.

Baumeister, Roy F. y Bushman, J. (2017), Social Psychology and Human Nature, Cengage Learning, Boston (MA).

Benedicto XVI (Ratzinger, Josef) (2005), Homilía 8 de diciembre de 2005, en https://www.vatican.va/content/benedict-xvi/es/homilies/2005/documents/hf_ben-xvi_hom_20051208_anniv-vat-council.html

Bergstrand, Jack (2013), "Good bye Productivity. Hello Engagement", en www.drucker.institute/thedx/good-bye-productivity-hello-engagement/ (cons. 25/01/2022).

Castillo, Genara (2015), "Aportes a la noción de liderazgo desde la filosofía de Leonardo Polo", en Sellés, Juan F., El hombre como solucionador de problemas. Investigaciones en torno a la antropología de Leonardo Polo, SPUN, pp. 275287.

Cornelius, Randolph R. (2006), "Magda Arnold's Thomistic Theory of Emotion, the Self-Ideal, and the Moral Dimension of Appraisal", Cognition and Emotion, v. 20, n. 7, pp. 976-1000.

Covey, Stephen R. y Collins, Jim (2013), The 7 babits of Higbly Effective People, $25^{\text {th}}$. Anniv. ed., Salt Lake City. 
Covey, Stephen R.; Merrill, Roger y Merrill, Rebecca (1994), First Things First, Simon and Schuster, Nueva York.

Csikszentmihalyi, Mihaly (1991), Flow: The Psychology of Optimal Experience, Harper Perennial, Nueva York.

Diener, Edward y Biswas-Diener, Robert (2008), Happiness: Unlocking the Mysteries of Psychological Wealth, Blackwell Publishing, Walden.

Doerr, John (2018), Measure what Matters, Penguin Books, Nueva York.

Fredrickson, Barbara (2013), "Positive Emotions Broaden and Build", Advances on Experimental Social Psychology, v. 47, pp. 1-54

- Fredrickson, Barbara (2018), "The Biological Underpinnings of Positive Emotions and Purpose”, en Baumeister, Roy F. y Forgas, Joseph P., (eds.) The Social Psychology of Living Well, Psychology Press, pp. 163-180.

Greenwood, Ronald G. (1981). "Management by Objectives: As Developed by Peter Drucker, Assisted by Harold Smiddy", The Academy of Management Review, 6, n. 2, pp. 225-230.

Izaguirre, Jesús M. y Moros, Enrique (2007), "La tarea del educador: la sindéresis", Studia Poliana vol. 9, pp. 103-127.

Johnson, Mark W. y Suskewicz, Josh (2020), Lead from the Future, Harvard Business Review Press, Cambridge (MA). Oxford.

Kristjánsson, Kristján (2018), Virtuous Emotions, Oxford University Press,

Mintzberg, Henry (2013), Simply Managing, Berrett-Koehler Publishers, San Francisco.

Mischel, Walter (2014), The Marshmallow Test, Transworld Publishers, Londres.

Peterson, Jordan B. (2018), 12 Rules for Life, Penguin Random House, Londres.

Polo, Leonardo (1998), "La esperanza”, en Scripta Theologica 30, n. 1, pp. 157-164

Polo, Leonardo (2015a), Introducción a la filosofía, Obras completas, Serie A, v. XII, EUNSA, Pamplona.

Polo, Leonardo (2015b), La originalidad de la concepción cristiana de la existencia, Obras completas, Serie A, v. XIII, EUNSA, Pamplona pp. 187-378. 
Polo, Leonardo (2016a), Quién es el hombre. Un espiritu en el tiempo, Obras completas, Serie A, v. X, pp. 23-211.

Polo, Leonardo (2016b), La esencia del hombre, Obras completas, Serie A, v. XXIII, EUNSA, Pamplona.

Polo, Leonardo (2017), Persona y libertad, Obras completas, Serie A, v. XIX, EUNSA, Pamplona.

Polo, Leonardo (2018), Ética. Hacia una versión moderna de los temas clásicos, Obras completas, Serie A, v. XI, pp. 139-312.

Polo, Leonardo (2019), Filosofía y economía, Obras completas, Serie A, v. XXV, EUNSA, Pamplona.

Polo, Leonardo (2019b), Ayudar a crecer, Obras completas, Serie A, XVIII, EUNSA, Pamplona, pp. 139-308.

Polo, Leonardo y Llano, Carlos (2019), Antropología de la acción directiva, en Polo, Leonardo, Obras completas, Serie A, XVIII, EUNSA, Pamplona, pp. 309-482.

Ribera, Albert y Ceja, Lucía (2017), "Flow: Flourishing at Work", en Mercado, Juan A. (Ed.), Personal Flourishing in Organizations, Springer, Cham, pp. 91-119. York.

Seligman, Martin E. P. (2003), Authentic Happiness, N. Brealey, Nueva

Tomasello, Michael (2019), Becoming Human. A Theory of Ontogeny, Harvard University Press, Cambridge (MA).

Zak, Paul (2013), Measurement Myopia, en www.drucker.institute/thedx/ measurement-myopia/ (cons. 25/01/2022) 
